\title{
Enquête au cœur des FabLabs, hackerspaces, makerspaces
}

Le dessin comme outil d'observation

\section{Camille Bosqué}

\section{(Q) OpenEdition \\ Journals}

\section{Édition électronique}

URL : https://journals.openedition.org/tc/7579

DOI : $10.4000 /$ tc. 7579

ISBN : 0248-6016

ISSN : 1952-420X

Éditeur

Éditions de l'EHESS

\section{Édition imprimée}

Date de publication : 24 décembre 2015

Pagination : 168-185

ISBN : 978-2-7132-2505-5

ISSN : 0248-6016

Référence électronique

Camille Bosqué, "Enquête au cœur des FabLabs, hackerspaces, makerspaces », Techniques \& Culture [En ligne], 64 | 2015, mis en ligne le 24 décembre 2018, consulté le 29 septembre 2022. URL : http:// journals.openedition.org/tc/7579; DOI : https://doi.org/10.4000/tc.7579 


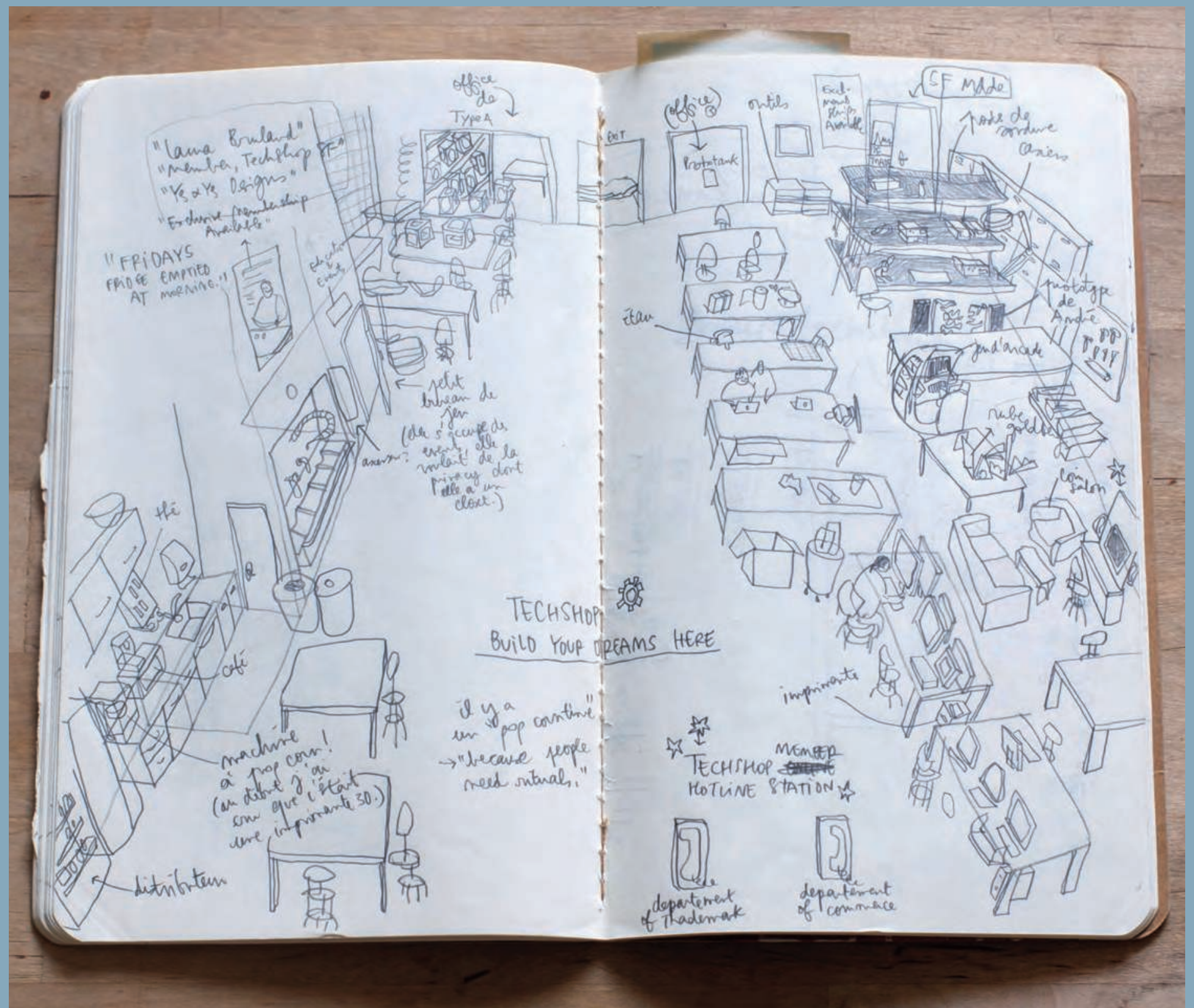




\section{Enquête au cœur des FabLabs, hackerspaces, makerspaces}

\section{Le dessin comme outil d'observation}

Le développement et la diffusion de machines de fabrication numérique à des prix de plus en plus accessibles ont permis au mouvement maker de se développer depuis les années 2000, d'abord aux États-Unis, puis à une échelle internationale.

Les makers ${ }^{1}$ se situent au croisement entre des ressources et méthodes qui se rapprochent de celles du design, de l'industrie et du prototypage, mais ils envisagent pour la plupart cette production selon des logiques qui relèvent davantage du hobby. Lapprentissage par la pratique, la décentralisation et le partage de compétences; l'autoproduction, l'accomplissement personnel par la fabrication et l'émancipation par les techniques numériques de fabrication sont les idées fortes portées par ce mouvement. L'éclosion de la culture maker a généré dans son sillage la multiplication de makerspaces ${ }^{2}$, espaces de fabrication numérique équipés de machines mises en commun. Ce terme générique qualifie des espaces aux généalogies aussi variées que les hackerspaces - dont l'existence précède l'avènement du mouvement maker - et les FabLabs ${ }^{3}$, dont le réseau se déploie dans le monde depuis les années 2000. Les hackerspaces existent depuis une trentaine d'années. Initialement plutôt tournés vers des activités de programmation informatique, ces espaces sont le lieu de pratiques qui dépassent le code pour envisager une approche libre du hack dans un sens élargi. En anglais, "to hack» signifie couper, tailler à la hache. Cela recoupe le détournement d'objet, la fabrication ou la réparation d'éléments techniques en lien avec une forme de création et d'expérimentation. Au-delà des réalisations concrètes, il est possible de tout hacker. Les modèles d'organisation sociale dans ces espaces collectifs peuvent donc aussi être l'objet d'une redéfinition. Ces espaces dédiés à la fabrication numérique se fondent sur des pratiques communes ou très proches qui défendent l'idée d'un travail singulier, libre et créateur. Les Fablabs peuvent être assimilés à ce que Ray Oldenburg appelle des «tiers-lieux» (1989). Selon la définition qu'il établit dans son étude, un tiers-lieu se définit comme un endroit (le plus souvent urbain) qui n'est ni tout à fait un domicile, ni 
totalement un lieu de travail. Cet espace mixte présente donc des caractéristiques qui relèvent autant de la sphère privée que de la sphère professionnelle. C'est un lieu neutre, libre d'accès et qui ne filtre pas a priori les individus selon des traits stigmatisants comme le niveau social, les compétences professionnelles ou les origines ethniques. Ces lieux, cafés, salons ou espaces collectifs ont pour rôle de favoriser la discussion et les interactions sociales.

Afin de comprendre la manière dont les idéaux et promesses portés par le mouvement maker s'incarnent dans ces espaces communs, j'ai mené pendant deux ans des enquêtes et observations de terrain. Designer de formation, engagée dans une thèse en esthétique, et de ce fait ethnologue «non assermentée», ma démarche de recherche se construit loin d'une étude statistique et en marge des catégories ou méthodes qualitatives éprouvées. Lors de mes différents séjours au cœur de ces communautés, j’ai pu ainsi expérimenter mes propres logiques d'observation et d'intégration, bricolant ou adaptant moi-même des tactiques particulières pour épouser au mieux les réalités des différents terrains observés.

En tant que designer, la fréquentation d'espaces dédiés à la fabrication et à la création a été l'occasion de tester les limites et opportunités de certaines stratégies d'observation participante. Ma pratique du terrain a été déterminée par une habitude prise assez tôt: dessiner, rester côte à côte avec les habitants des ateliers où je me suis rendue. Eux bricolent, je dessine. J'écoute. Je discute. Je suis là. Le rapport à l'autre instauré par la présence du carnet de croquis entre mes informateurs et moi en a fait un instrument de travail précieux. Pendant mes heures passées sur le terrain, j'ai confondu dans mes carnets des notes prises sur le vif avec des croquis de l'espace, des représentations de certains détails ou certaines scènes attrapées rapidement. On y trouve également quelques portraits ou des schémas d'explications techniques, des anecdotes qui me permettent a posteriori de reprendre par la description écrite certains moments marquants, parfois complétés d'enregistrements audio. Les images imparfaites ou inachevées qui peuplent mes carnets accompagnent la narration de mon expérience ethnographique. En présentant ici des pages directement extraites de mes carnets d'enquête, j'affirme l'importance peu étudiée d'une pratique de l'enquête par, avec et autour du dessin. Comme le démontre Michael Taussig, le croquis a des valeurs de captation qui n'ont rien à voir avec celles de la photographie: «photographier c'est prendre, dessiner c'est faire» (Taussig 2011 : 213). Dessiner revient à comprendre, interroger, faire parler, pousser à décrire et à dire. Un dessin n'est pas une image fixe et distante, il est une perception intime et personnelle qui rend visible un point de vue particulier.

Je fais l'hypothèse qu'en tant que technique empirique, le dessin comme instrument de travail permet de provoquer une forme d'empathie fertile, c'est-à-dire de contourner les risques d'une fusion affective avec le terrain d'observation tout en instituant une communication plus directe avec les «autochtones» qui l'habitent. Il ne s'agit pas de décrire simplement ce qui se passe dans ces fameux FabLabs, hackerspaces ou makerspaces, mais aussi de les examiner indirectement, en s'attachant à qualifier le rapport établi avec mes «informateurs» par la présence du carnet de croquis et les conduites que cela a pu entraîner. Ce qui détermine le recours au dessin est lié au fait de vouloir rester longtemps dans les lieux visités sans nécessairement interrompre ou m’insérer dans les activités en cours. Sur des terrains plus courts, le dessin s'impose également 
comme moyen - ou prétexte - pour rester présente sans enclencher le processus directif des entretiens auprès des personnes qui sont là. C'est une manière de déjouer l'ennui tout en faisant accepter ma présence sur une longue plage de temps, sans pour autant entrer frontalement en conversation avec les habitants de l'endroit. Mais je n'avais pas anticipé la valeur d'attraction de cette pratique auprès des communautés dans lesquelles je me suis installée. À Noisebridge-après avoir passé de longues heures à simplement varier mes points de vue pour saisir par le crayon les aménagements, objets, machines, fragments d'éléments ou inscriptions murales - mon activité silencieuse a fini par attirer la curiosité de quelques habitants de l'endroit, qui se sont penché sur mon carnet pour commenter mon travail, s'étonnant de mes choix de points de vue pour représenter l'espace: "You're hacking the perspective, it's genius ${ }^{4}$ !». S'ensuivirent quelques remarques sur la place de telle ou telle chose dans l'image, des explications sur la présence de cet outil ou de cette boîte dans ce coin précis, ou des révélations sur certains éléments présents dans mon dessin mais dont la fonction m'était justement restée mystérieuse. Il s'agit bien ici d'établir une distance, d'objectiver, de représenter, mais aussi de tromper cette asymétrie entre l'observateur et l'observé par un bricolage ouvert, qui détourne l'attention, efface le cadre artificiel de l'enquête et lisse le fossé entre celui qui observe pour comprendre, et le sujet.

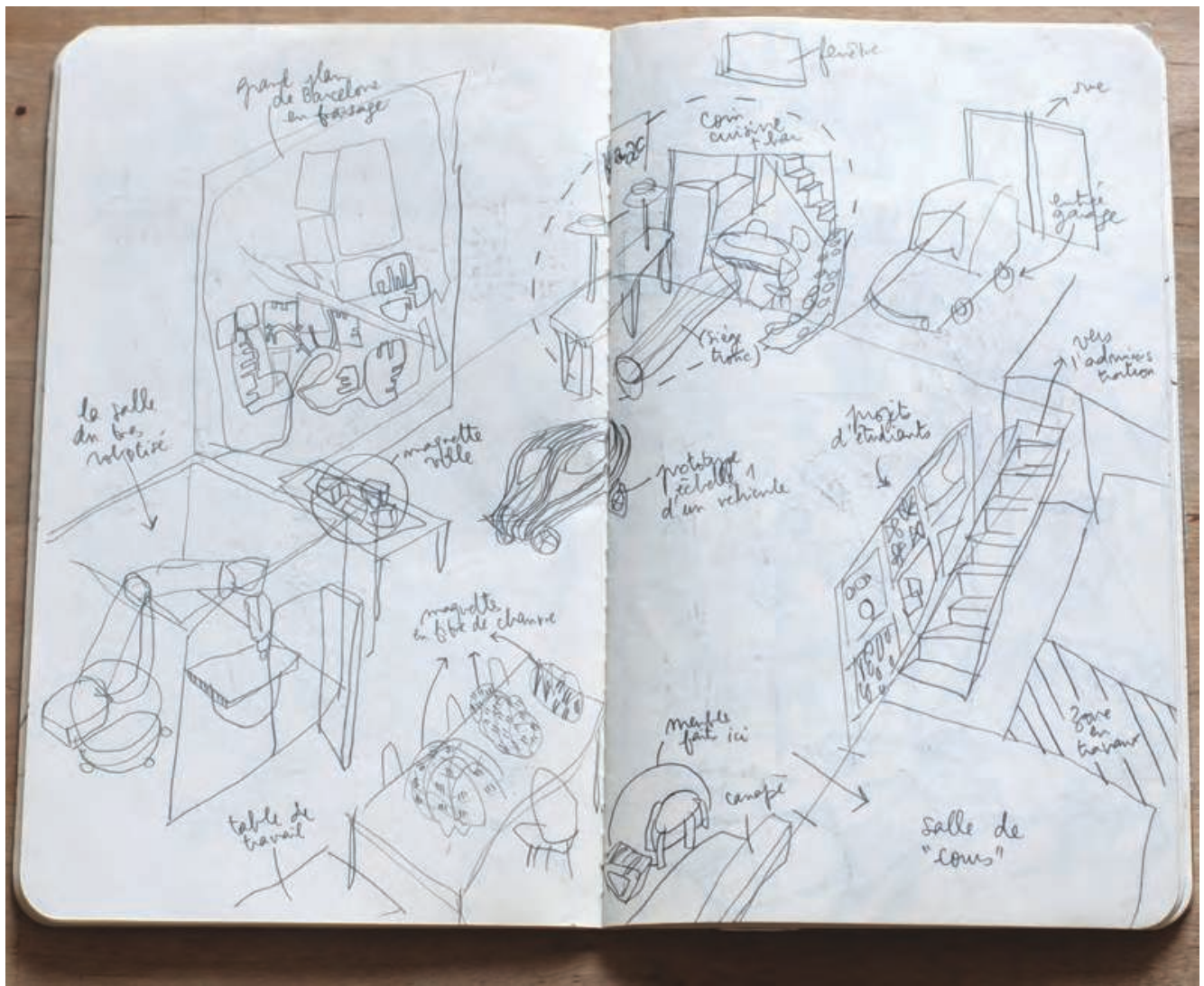

Le FabLab IAAC de Barcelone est l'un des plus actifs en Europe. Il est installé dans une école d'architecture. A l'entrée, comme dans de nombreux espaces de fabrication, des projets sont exposés. On y trouve des prototypes et maquettes qui font la démonstration des possibilités offertes par les différentes machines présentes dans l'atelier: imprimantes 3D, fraiseuse numérique, découpeuse laser, etc. 


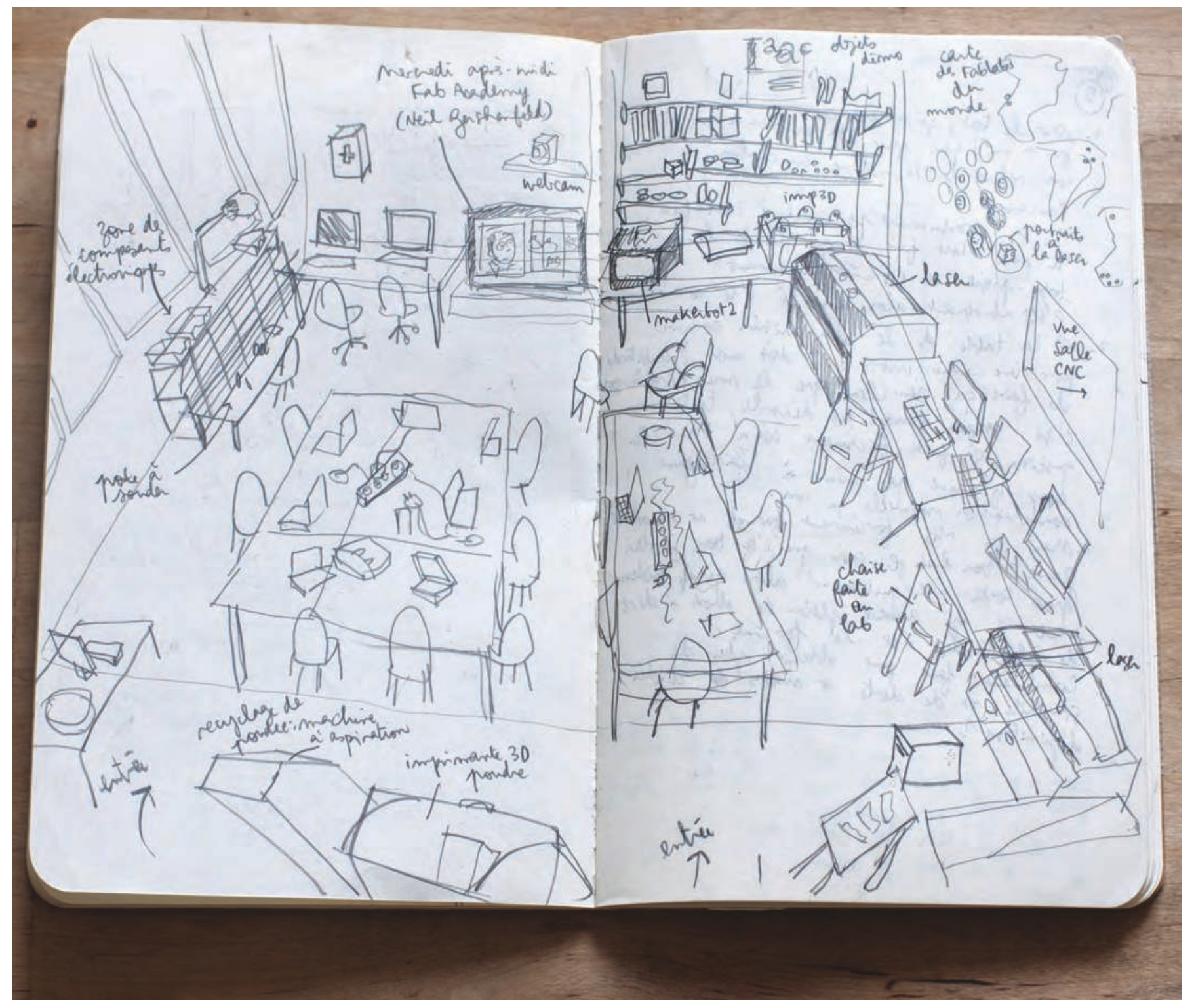

2. Une salle à part est réservée aux cours ou aux activités plus silencieuses et moins salissantes. Quelques livres côtoient des petites imprimantes 3D, des composants électroniques et des postes à souder. Un grand écran de télévision permet aux étudiants qui suivent la FabAcademy, un programme de cours par visioconférence, de communiquer avec d'autres FabLabs dans le monde sur les projets en cours. Le réseau international des FabLabs est représenté sur le mur de droite, par des épingles sur une grande carte du monde. 

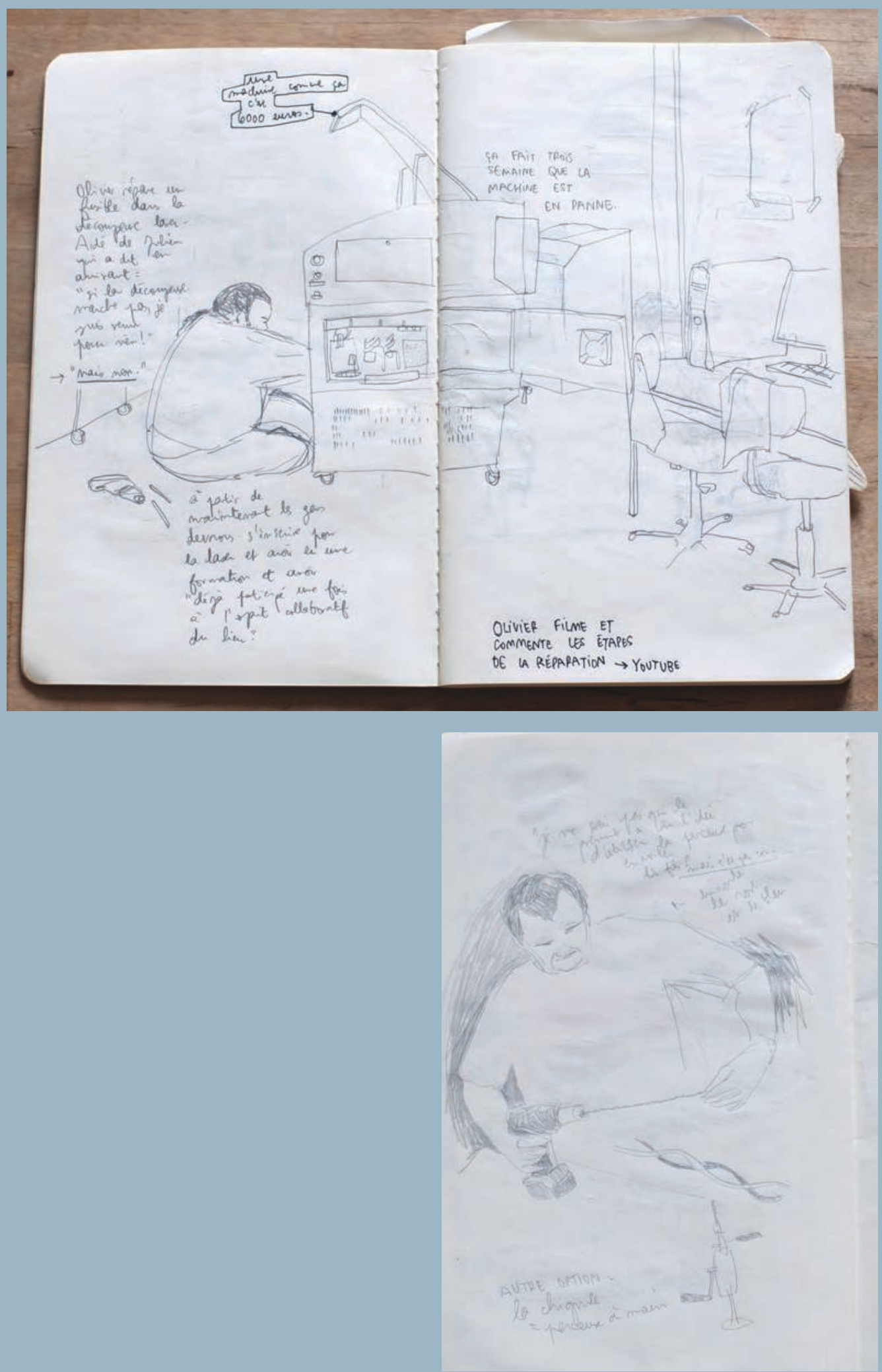

3. Au FacLab à Cergy-Pontoise, Olivier répare la découpeuse laser. Quand j'ai rencontré les cofondateurs du FacLab et que je leur ai expliqué que je souhaitais m'y installer pour quatre mois d'observation dans le cadre de ma thèse, on m'a invitée à me plier aux règles de vie établies pour l'atelier: «Ici, on n'aime pas les observateurs. » Les règles à respecter tiennent en trois mots: " participer, partager, documenter ». Cette devise est affichée dans le FacLab à côté de la charte officielle du MIT. Le FacLab, situé dans l'université de CergyPontoise à Gennevilliers, est l'un des FabLabs français les plus actifs. Malgré son implantation dans l'université, il est essentiellement fréquenté par des personnes extérieures à la faculté, sorte d'excroissance à la marge des règles classiques de l'institution où se croisent retraités, auto-entrepreneurs, jeunes curieux, designers ou bricoleurs.
Pour enrouler ensemble deux fils électriques, Michel utilise une perceuse. Les pratiques de réparation ou de détournement sont essentielles dans l'économie du mouvement maker. Ainsi, quand une machine de l'atelier tombe en panne, le premier réflexe n'est pas d'appeler le service après-vente mais d'ouvrir soi-même le capot de l'appareil pour ajuster les réglages et la remettre en route. Documenter ces gestes fait partie des réflexes défendus par ces bricoleurs augmentés par les technologies numériques. 


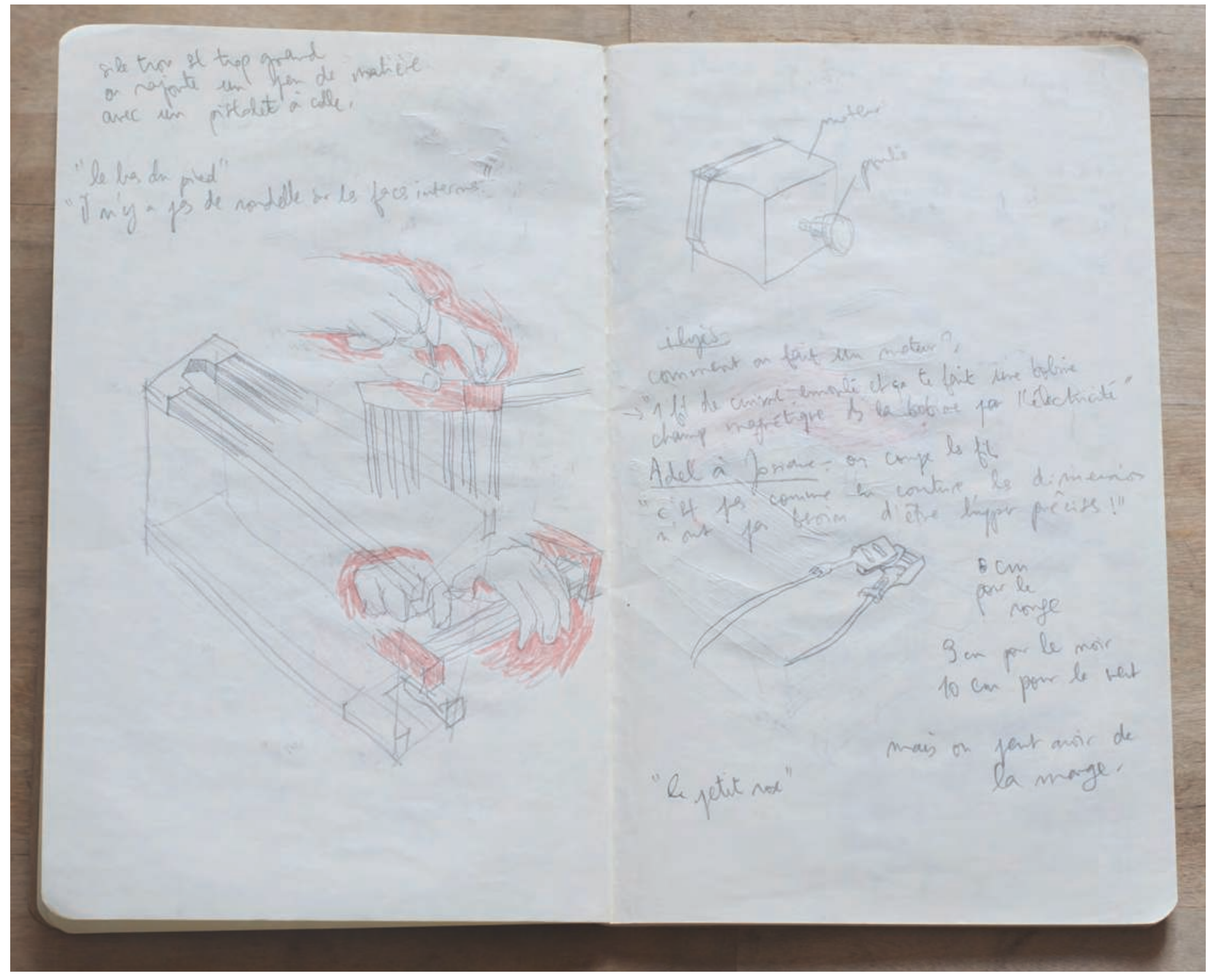

5 et 6. L'importance de faire ensemble:

«Là, on ajuste la plaque chauffante.... 


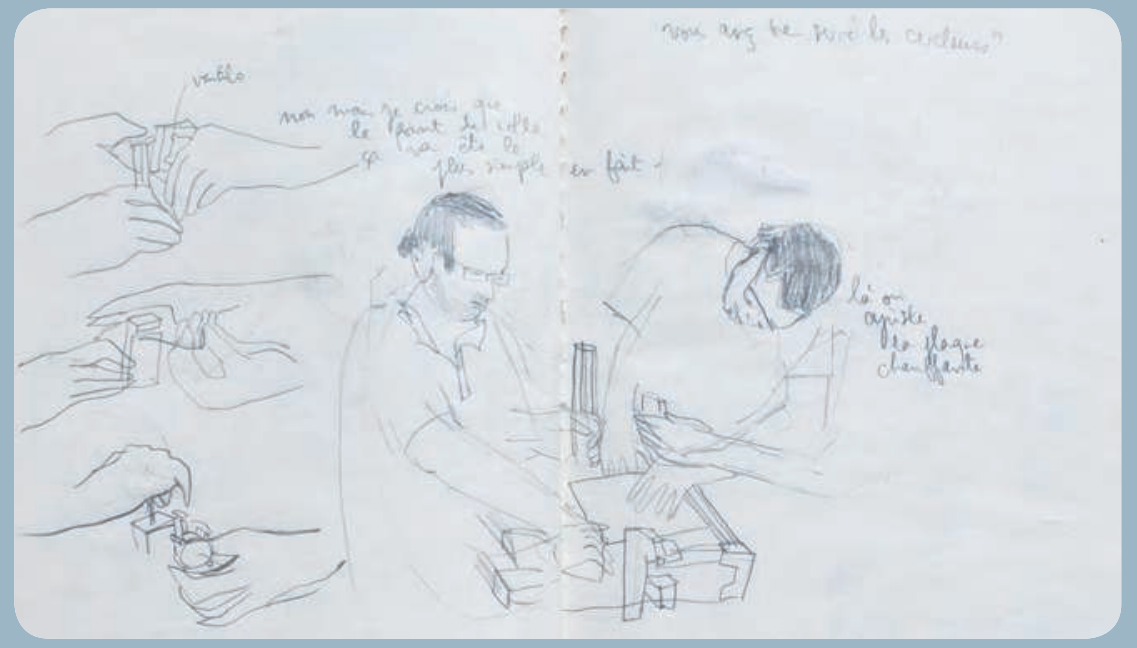

6 et 7 . Les imprimantes 3D open source, comme la célèbre RepRap, sont présentes dans la plupart des espaces collectifs de fabrication. Au-delà de l'utilisation classique de ces machines pour donner forme à de petits objets, construire une imprimante $3 \mathrm{D}$ est souvent un projet à part entière, qui ras semble plusieurs personnes et sert de prétexte à un échange de connaissances.

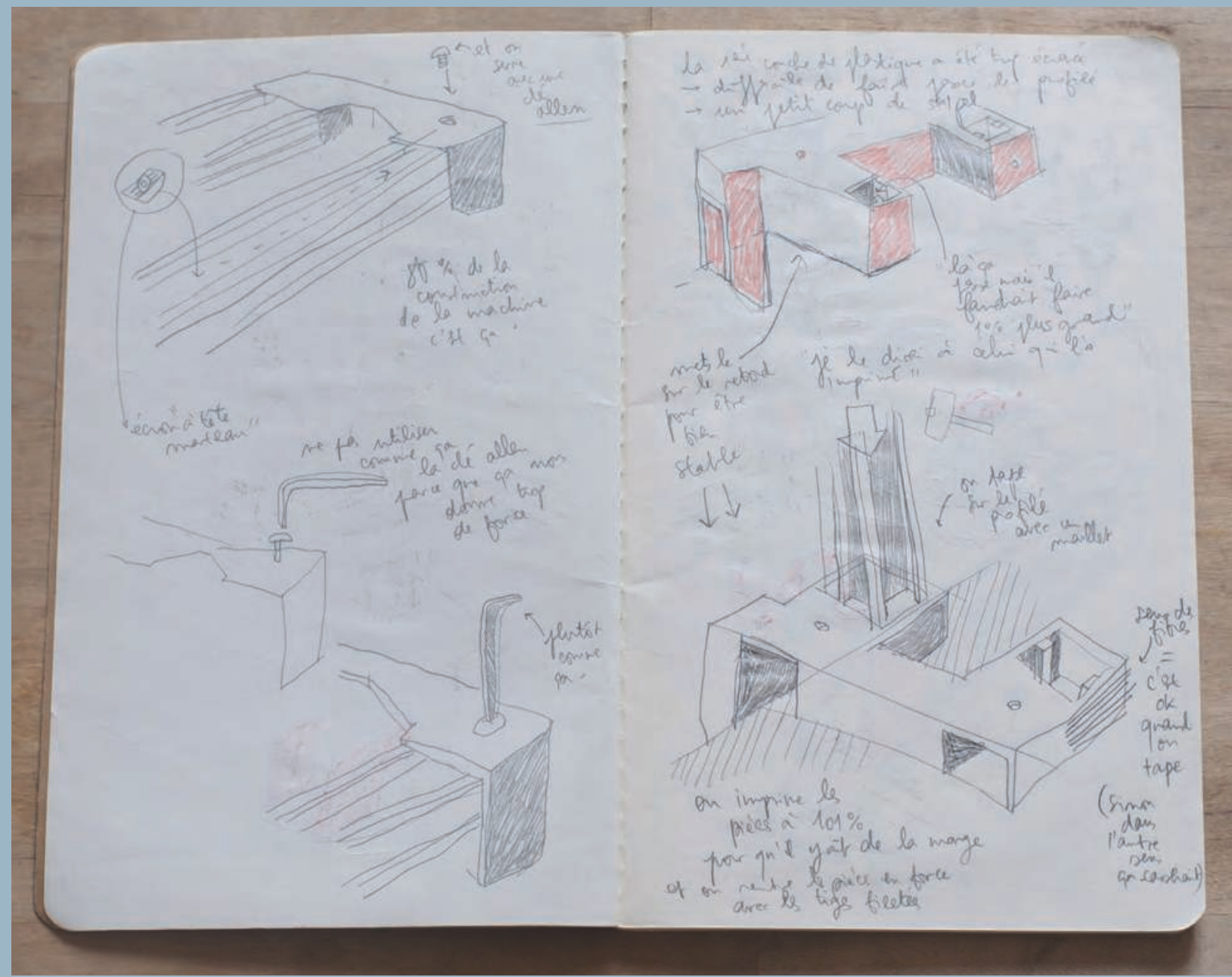




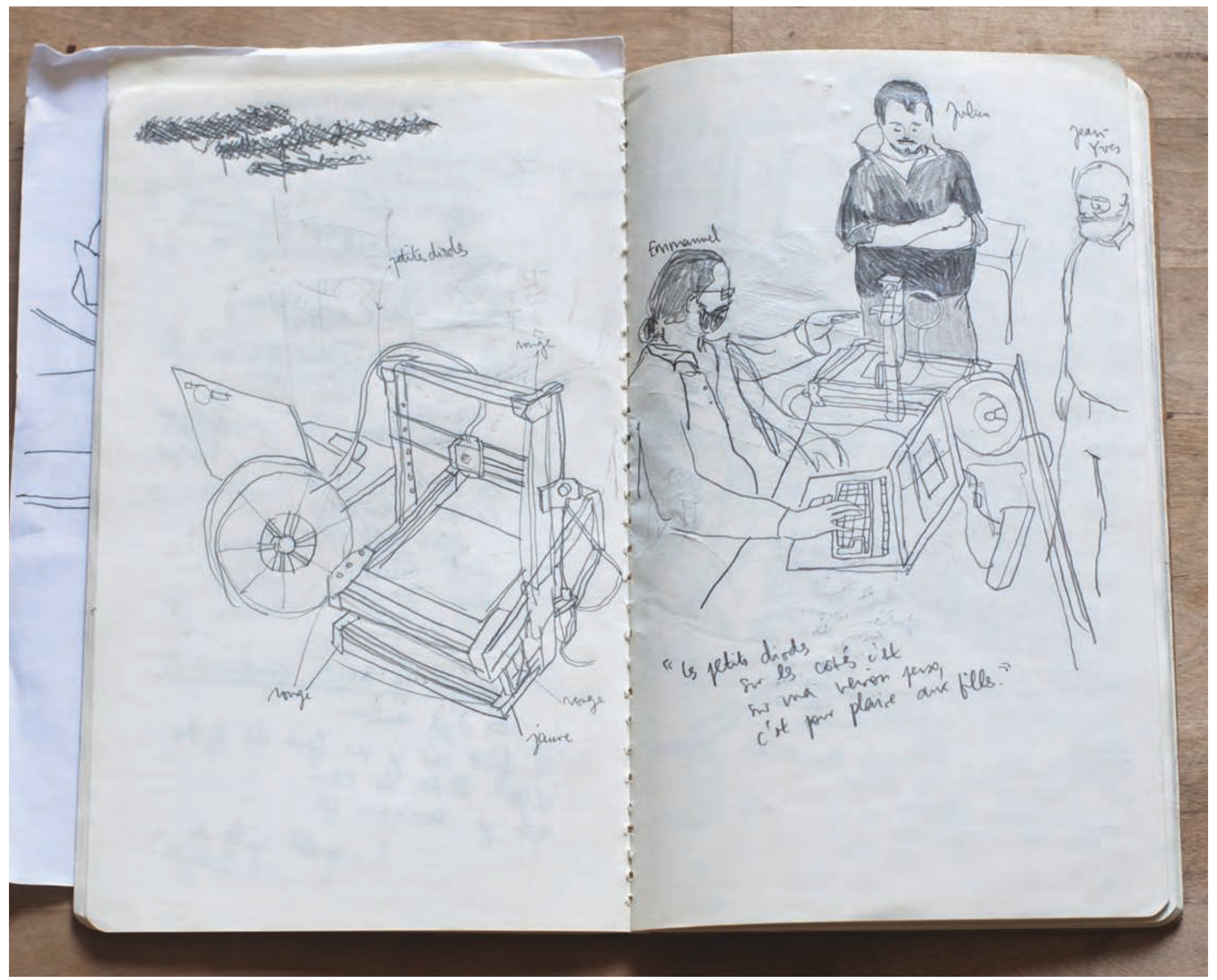

8. «Les petites diodes, c'est pour plaire aux filles »:

Emmanuel montre son imprimante $3 \mathrm{D}$ à Julien et Jean-Yves, au FacLab.

Pousser la porte de ces makerspaces et aller à la rencontre des communautés qui l'animent permet de faire rapidement ce constat simple: si ces espaces sont a priori «ouverts à tous», ils rassemblent beaucoup plus d'hommes que de femmes. 


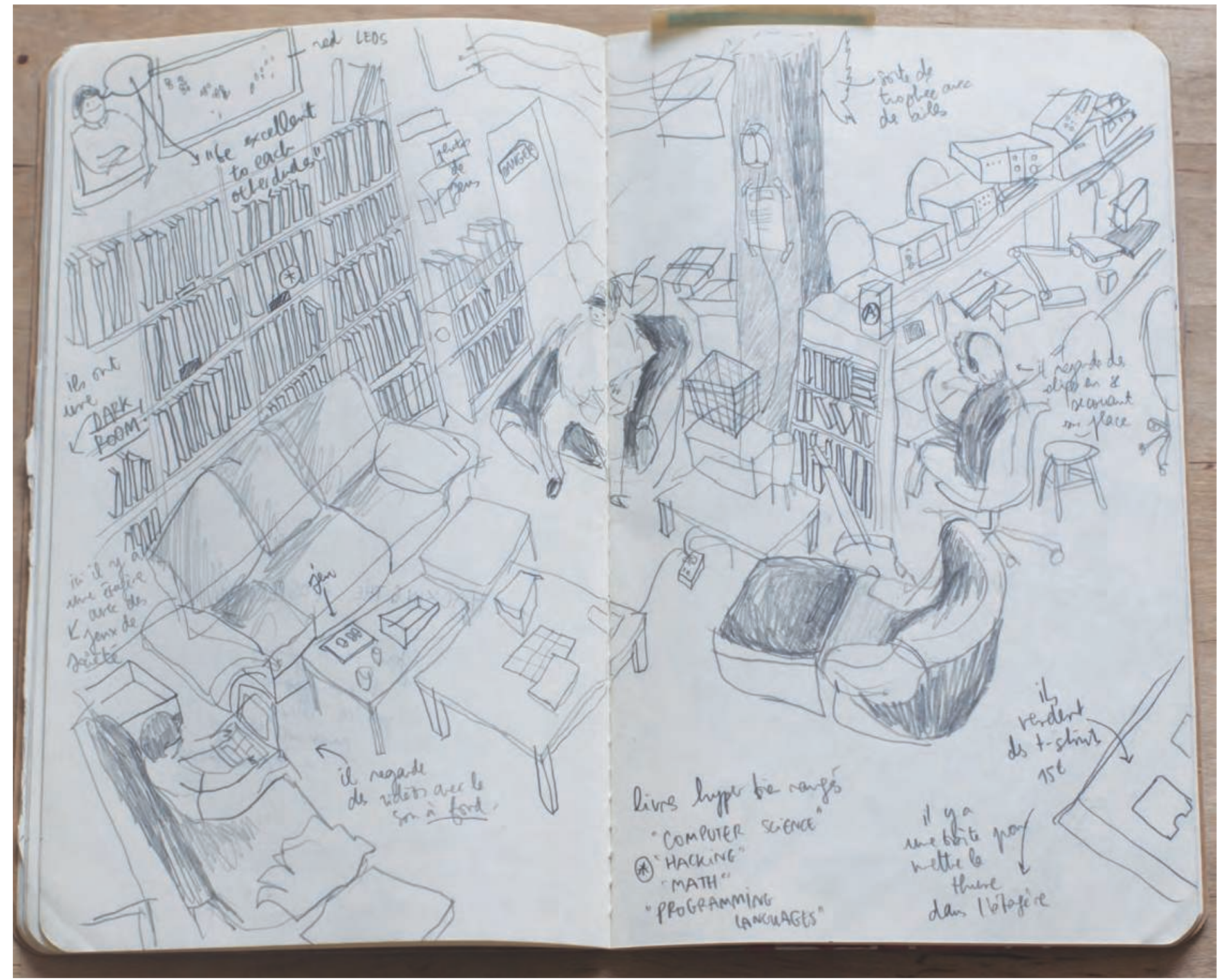

9. «40 \% of these don't work5». Le coin bibliothèque du hackerspace Noisebridge, à San Francisco.

Noisebridge existe au cœur du quartier hispanique de San Francisco depuis une dizaine d'années et revendique une philosophie d'inspiration anarchiste. Ce hackerspace se prête bien à une observation scrupuleuse: c'est un immense bricà-brac de machines en grande partie récupérées, de livres, de meubles hétéroclites, d'outils, de matériaux et d'affiches. Lieu de résistance et de contre-culture, Noisebridge est un lieu autogéré, dans l'esprit libertaire, où une seule règle - à interprétations variables - garantit le bon fonctionnement de la vie en communauté: «Be excellent to each other ${ }^{6}$.» Un autre slogan inscrit sur quelques affiches est appliqué assez spontanément par les usagers de Noisebridge: «Shut up and hack?.» 


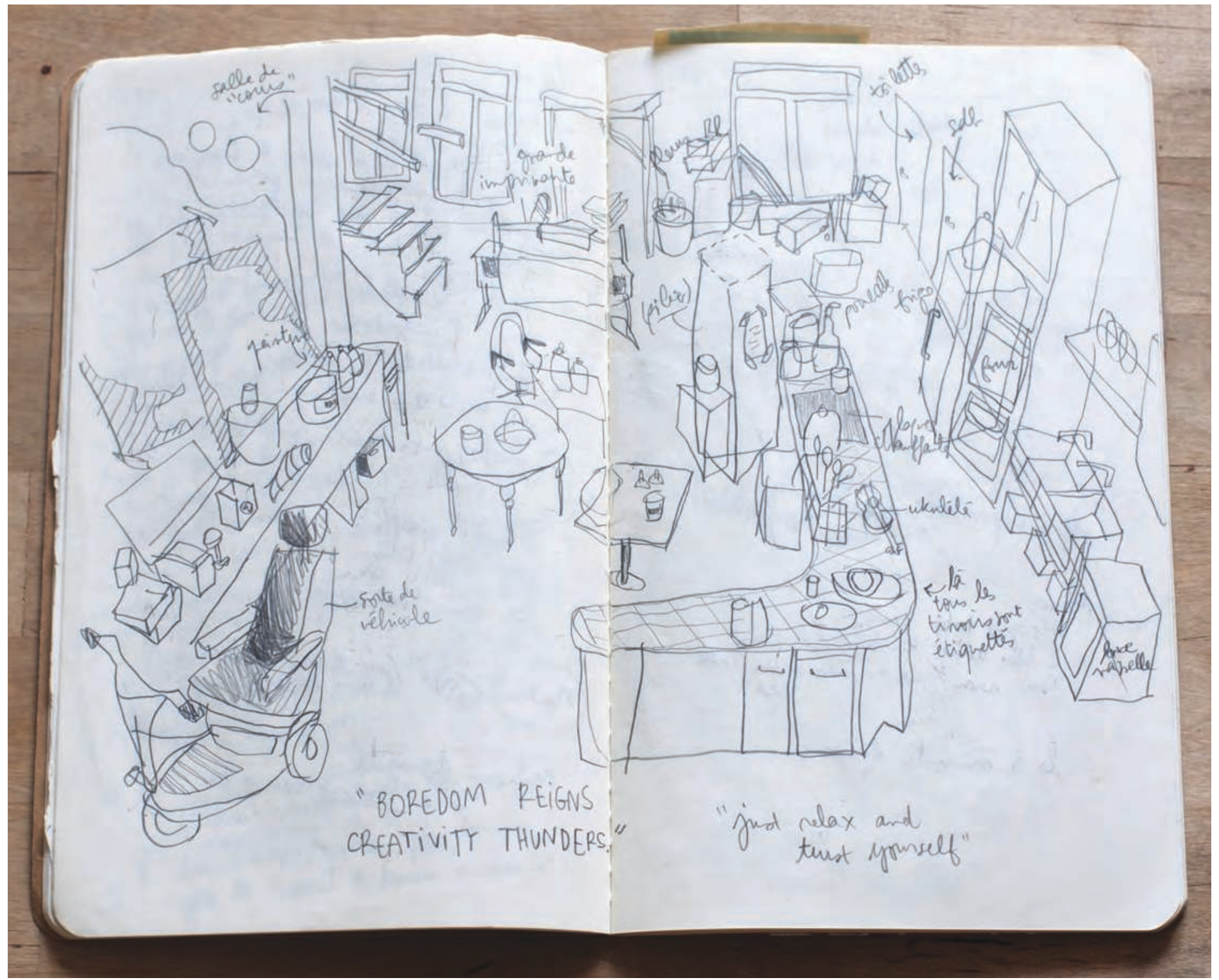

10. Le coin cuisine de Noisebridge: «Just relax and trust yourself ${ }^{8}$.»

Dans de nombreux hackerspaces ou makerspaces, la machine à café est souvent présentée comme une machine parmi d'autres, qui a autant - sinon plus - d'importance que le canapé dans lequel de nombreuses discussions s'engagent, un peu à l'écart des tables de travail. Selon Ray Oldenburg (1989), «la conversation est l'une des activités cardinales des tiers-lieux» qui garantit et maintient le plaisir d'être ensemble. De la même manière que certains cafés semblent être davantage conçus comme un lieu de socialisation plutôt que de pure consommation, certains ateliers partagés, comme ici Noisebridge, aménagent leurs espaces et organisent leurs moments de vie en privilégiant souvent la convivialité sur l'efficacité ou la fonctionnalité. II ne s'agit pas de venir sur place pour être performant et pour « produire» mais davantage pour goûter le plaisir de «faire à plusieurs », côte à côte. 


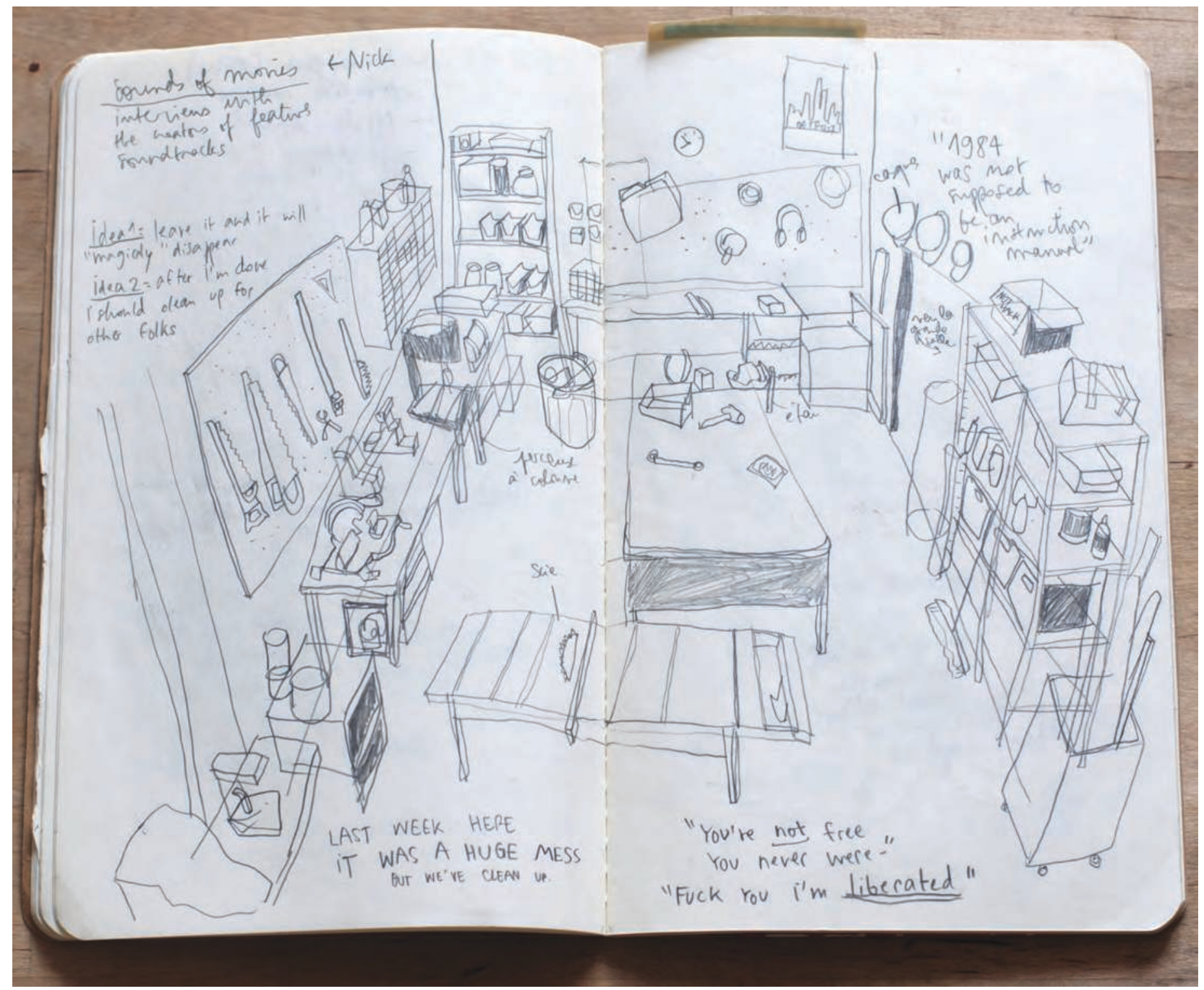

\section{. L'atelier bois de Noisebridge.}

A Noisebridge comme ailleurs, le bidouillage électronique, les lignes de code et les machines numériques n'ont pas entièrement remplacé les outils plus traditionnels, qui ont encore une place de choix. 


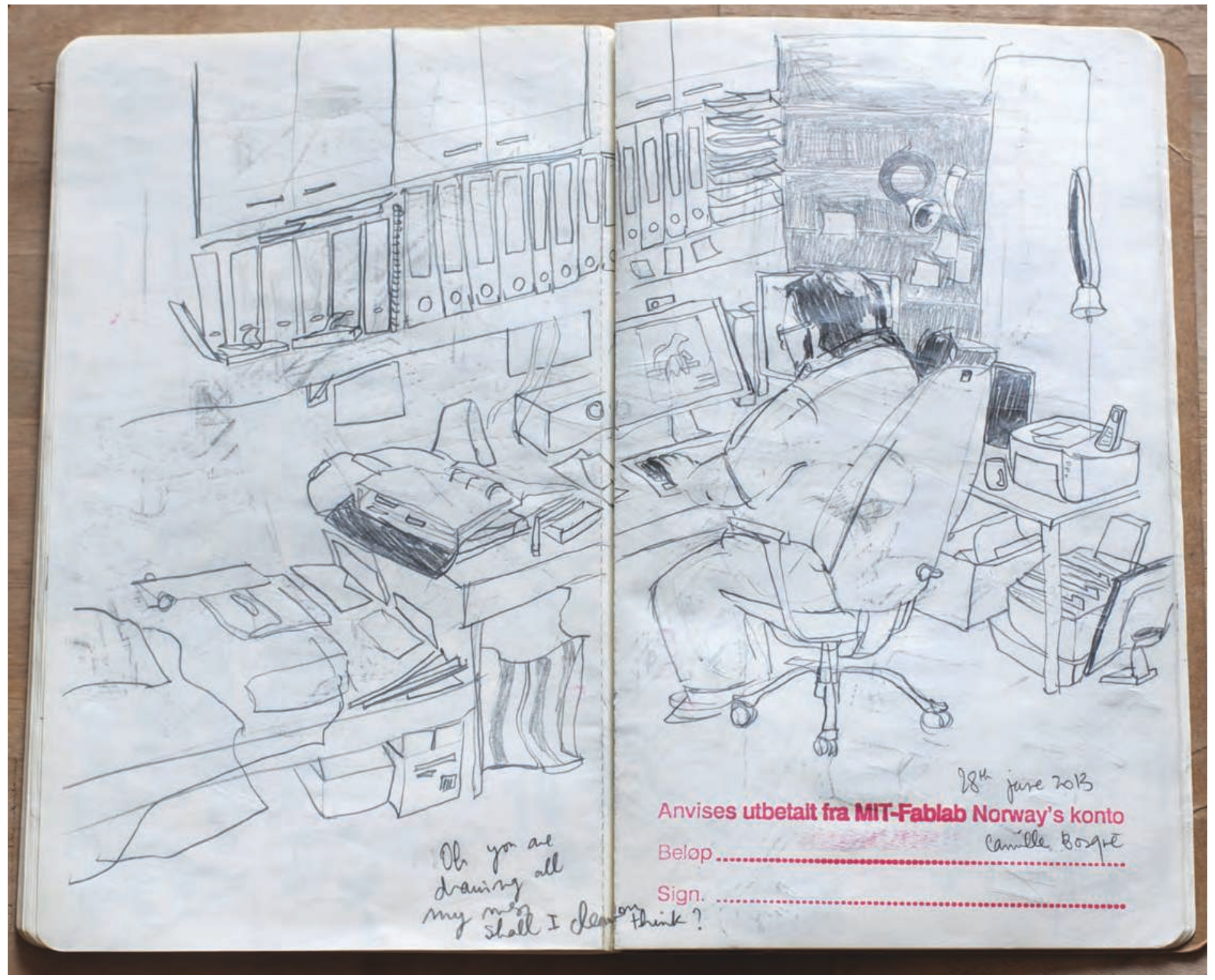

12. «Oh, you're drawing all my mess 9 !», s'écrie Haakon Karlsen.

Rejoindre le MIT-FabLab Norway nécessite un long voyage par étapes, depuis Oslo jusqu'à Tromsø et enfin Lyngen. C'est un des premiers FabLabs du monde, installé dans un immense chalet en longueur, au bord d'un fjord. 


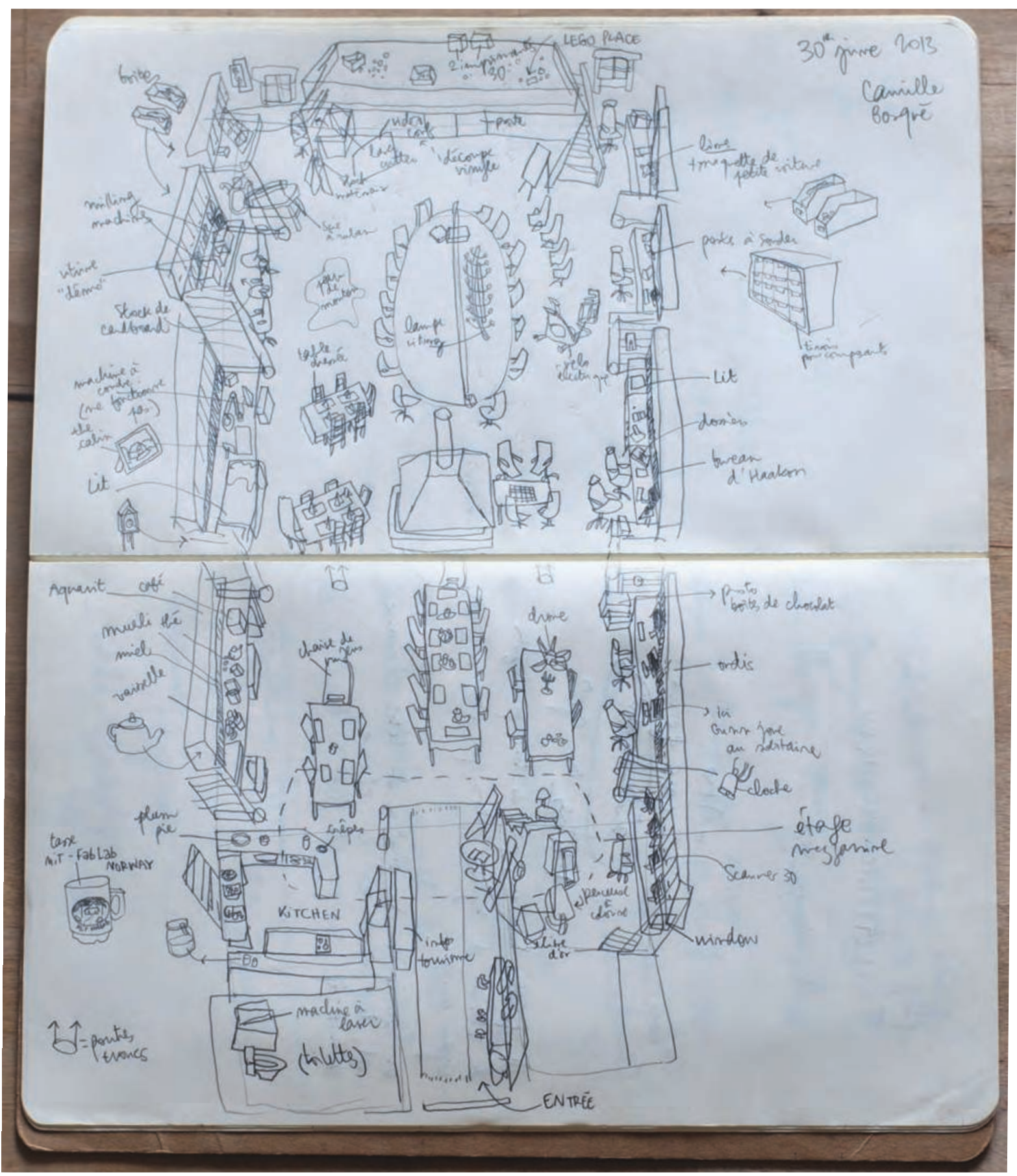

13. Plan général du MIT-FabLab Norway.

Haakon Karlsen estime qu'aujourd'hui son FabLab est davantage un community center ${ }^{\circ}$ qu'un lieu de prototypage: «On y a même déjà célébré un mariage! » Dans l'immense chalet, les machines, tables et postes de travail ont été installés sur tous les côtés de la grande pièce, le long des murs: postes à souder, découpeuse laser, imprimantes 3D, thermoformeuse, découpeuse vinyle, etc. Entre les bureaux et étagères, quelques lits sont installés, recouverts de fourrures et d'oreillers. Au centre, à côté de la grande table de réunion et de visioconférence, une énorme cheminée, plusieurs tables pour les repas et quelques fauteuils et tables basses occupent l'espace. 


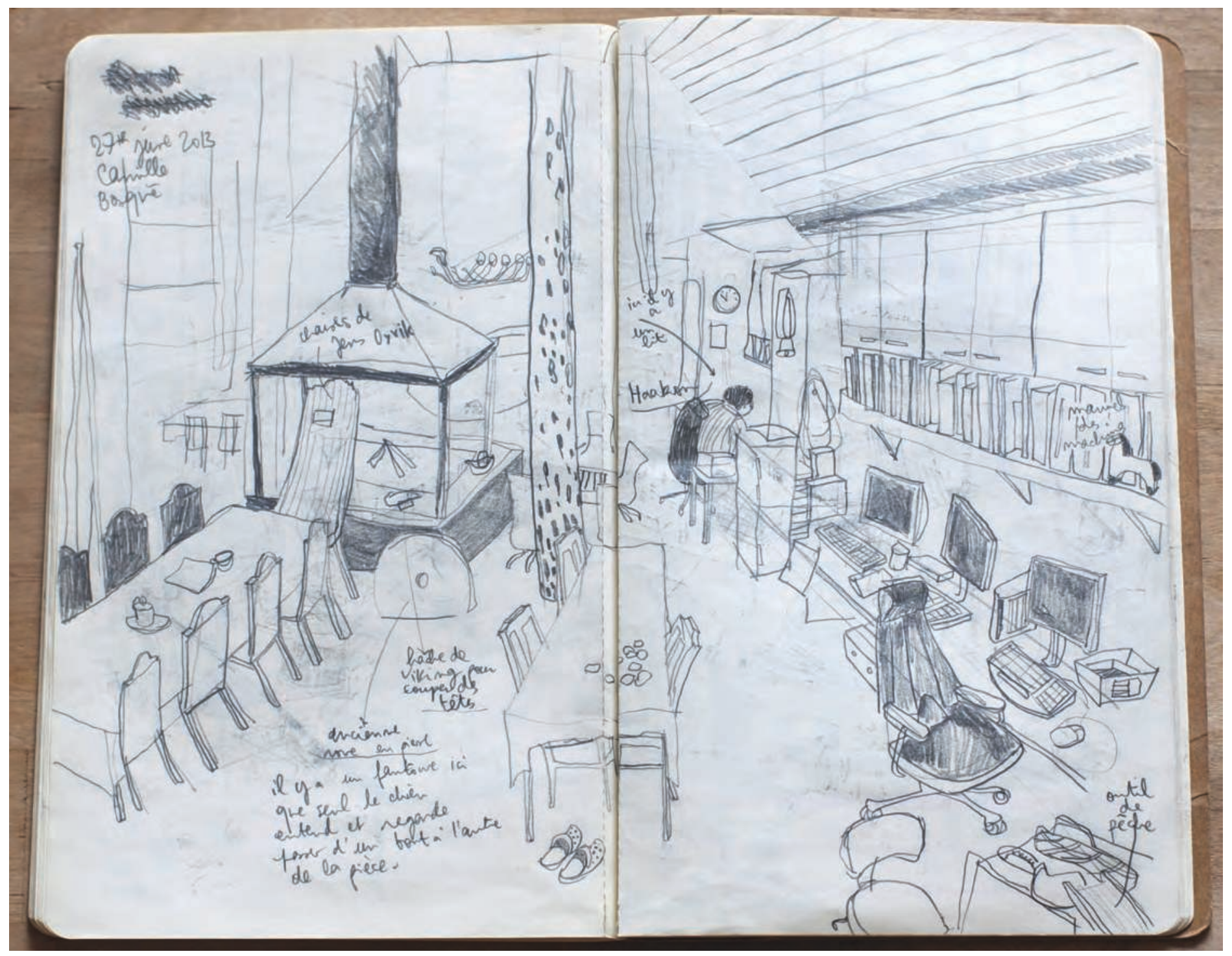

14. La cheminée du MIT-FabLab Norway. 


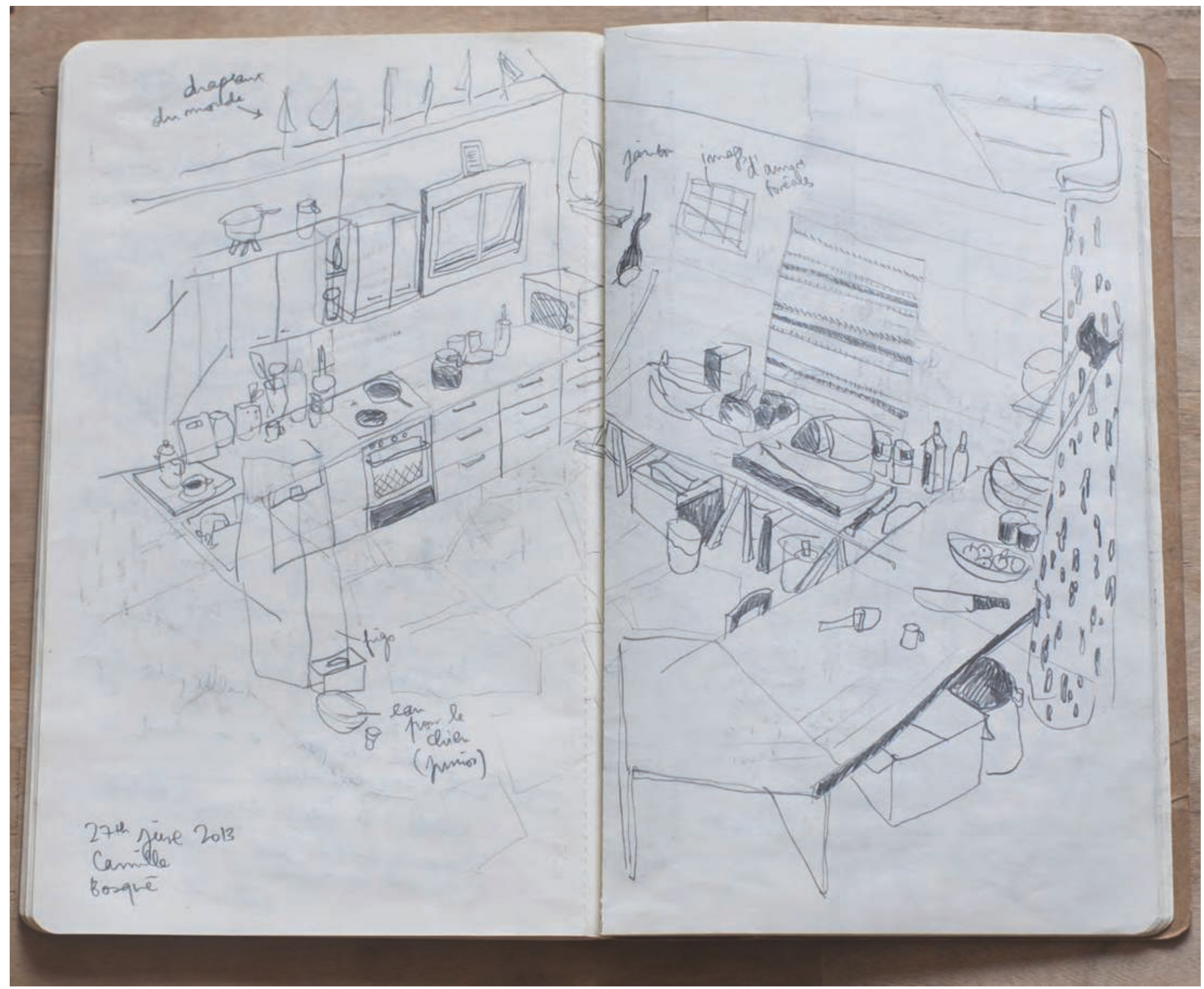

\section{La cuisine ouverte du MIT-FabLab Norway.}

Ces tables sont là pour accueillir à tout moment les visiteurs qui voudraient s'établir dans la région quelques jours en pension complète, pour faire de la randonnée ou d'autres activités de plein air. En effet, le MIT-FabLab Norway est depuis plusieurs années autant un gîte qu'un lieu de prototypage et de fabrication. Ce modèle hybride inattendu garantit une bonne partie de son financement. 


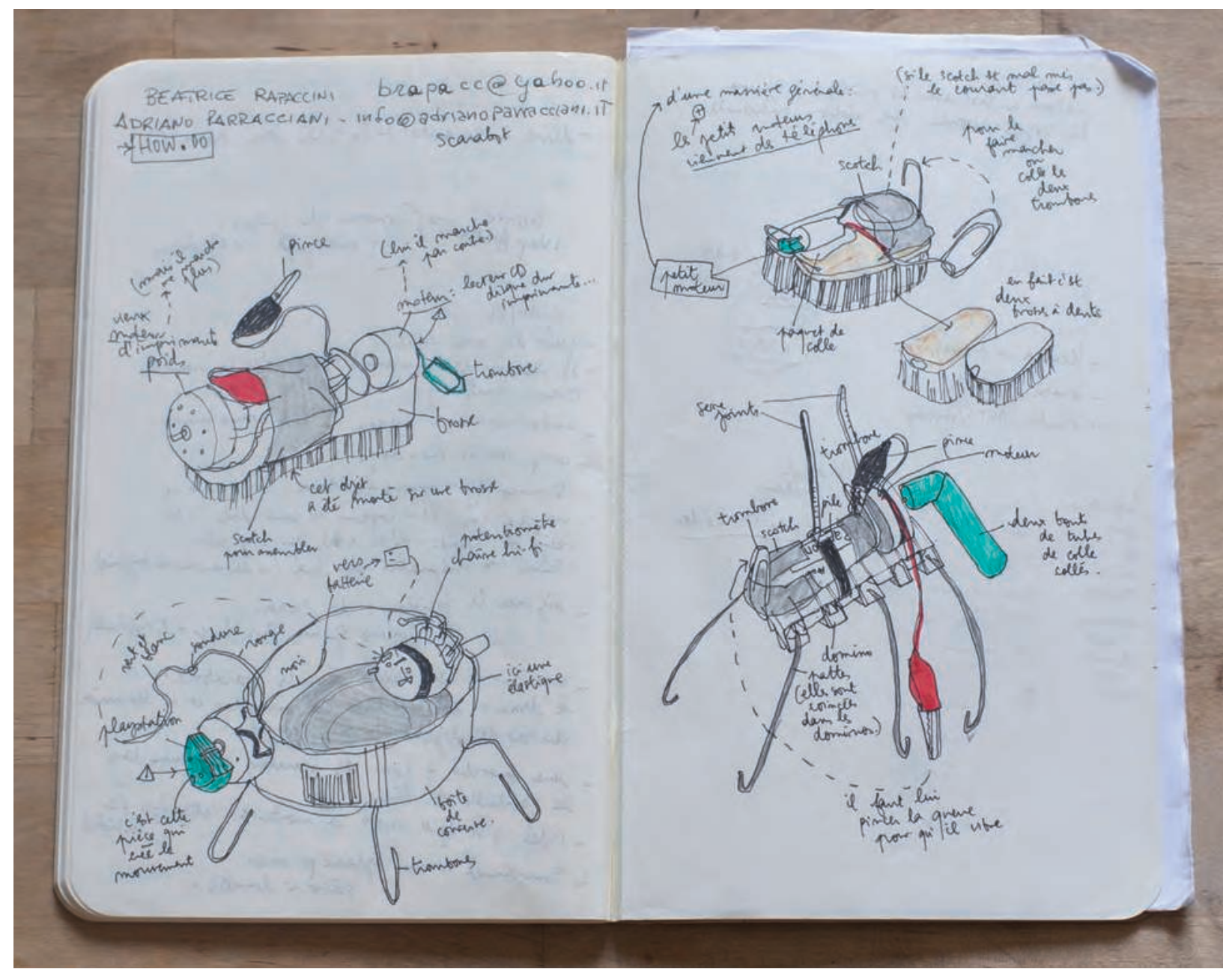

\section{Dessins de «Scarabots», à la Maker Faire.}

Les Scarabots sont composés de plusieurs éléments, qui sont associés à une pile électrique et à un petit vibreur récupéré sur de vieux téléphones portables. En connectant le fil rouge et le fil noir, la petite bête vibre et s'anime.

J'ai souvent cherché à provoquer les commentaires sur mon travail pour annoter mes dessins, comme lors de la Maker Faire de Rome en octobre 2013, où j'ai passé une après-midi entière à dessiner ces assemblages bizarroïdes conçus par un petit groupe d'enfants. Ils m'ont été décrits un à un: «ici j'ai mis un point de colle, ici c'est un trombone récupéré, là j'ai eu l'idée de mettre un entonnoir...»

17. A mon croquis initial s'ajoutent des annotations supplémentaires - précisions en palimpseste que les personnes observées tiennent parfois à faire apparaître parmi mes notes. Puisque je dessine, je reste longtemps assise à la même table que ceux avec qui je partage du temps. Nous bricolons côte à côte.

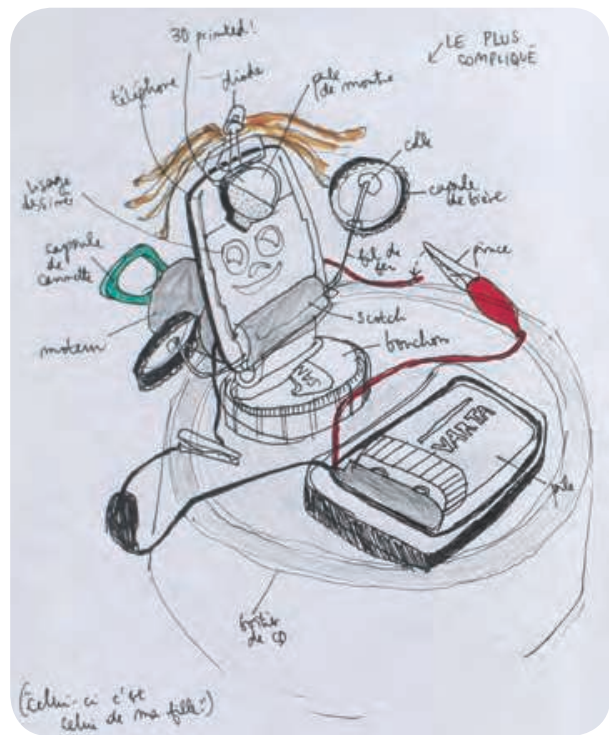




\section{Notes}

1. Un maker est un bricoleur augmenté par les nouvelles technologies.

2. Makerspace et un terme générique qui regroupe tous les ateliers collectifs de fabrication équipés d'outils traditionnels et de machines numériques.

3. FabLab est une contraction de l'anglais fabrication laboratory qui signifie «laboratoire de fabrication».

4. «Tu hackes la perspective, c'est génial!»

5. « $40 \%$ de tout cela est cassé.»

6. «Sois excellent avec ton prochain.»

7. «Tais-toi et hack. »

8. «Détends-toi et fais-toi confiance.»

9. «Oh, tu dessines tout mon désordre!»

10. Un community center est un lieu de vie collective, un espace dédié aux rassemblements, réunions, fêtes ou débats publics.

\section{I'auteure}

Camille Bosqué est une ancienne élève du département design de l'École normale supérieure de Cachan, diplômée de l'École Boulle et agrégée d'arts appliqués. Elle mène depuis 2012 une thèse en esthétique et design à l'université Rennes 2 et l'Ensci - Les Ateliers. Ses recherches portent sur la fabrication numérique personnelle dans les FabLabs, hackerspaces, makerspaces.

\section{Iconographie}

Image d'ouverture. TechShop : vue générale du premier étage, San Francisco.

Crédits pour les dessins et les photographies: (C) Camille Bosqué.

\section{Références}

Bosqué, C. \& Ricard, L. 2015 FabLabs, etc. Les nouveaux espaces de fabrication numérique, Paris: Eyrolles.

Oldenburg, R. 1989 The Great Good Place: Cafes, Coffee Shops, Community Centers, Beauty Parlors, General

Stores, Bars, Hangouts, and How They Get You Through the Day, New York: Paragon House.

Taussig, M. 2011 I Swear I Saw This, Drawings in Fieldwork Notebooks, Namely my Own, Chicago: University of Chicago Press.

\section{Pour citer cet article}

Bosqué, C. 2015 «Enquête au cœur des FabLabs, hackerspaces, makerspaces. Le dessin comme outil d'observation », TechniquesE Culture nº 64 «Essais de bricologie. Ethnologie de l'art et du design contemporains », p. 168-185. 